\title{
Agequake II: psychische Gesundheit älterer inhaftierter Personen - eine schweizweite Studie
}

\author{
Helene Seaward ${ }^{1}$ Laudelina Taboas Hidalgo ${ }^{1}$ Tenzin Wangmo ${ }^{1}$ Bernice S. Elger ${ }^{1,2}$ \\ Eingegangen: 6. August 2021 / Angenommen: 13. September 2021 / Online publiziert: 7. Oktober 2021 \\ (c) Der/die Autor(en) 2021
}

\section{Zusammenfassung}

Das Projekt Agequake II erhob Daten zur psychischen Gesundheit älterer inhaftierter Personen in der Schweiz, evaluierte die aktuelle Situation deren psychiatrischer Versorgung und untersuchte Optionen zu ethischen, rechtlichen und wirtschaftlichen Lösungen in Bezug auf altersbedingte Probleme. Agequake, benannt nach dem Phänomen der rapide wachsenden Anzahl älterer inhaftierter Personen, ist somit eines der wenigen Forschungsprojekte, das Daten zu der älteren Gefängnispopulation liefert und Ergebnisse zur Erweiterung des Wissens über diese wenig erforschte Gruppe beisteuert. Die Ergebnisse tragen zum besseren Verständnis der Lebensrealitäten gefangener älterer Personen bei, bieten Strategien zur Verbesserung derer physischer und psychischer Gesundheitsversorgung und zur Verringerung der Belastung des Gefängnispersonals und der größeren Öffentlichkeit.

Schlüsselwörter Alt $\cdot$ Haft $\cdot$ Maßnahme $\cdot$ Psychiatrie $\cdot$ Therapie $\cdot$ Gefängnis

\section{Agequake II: mental health of older incarcerated persons-Findings from Switzerland}

\begin{abstract}
The project "Agequake II" collected data on the mental health of older incarcerated persons in Switzerland, evaluated the current situation of their mental health care, and explored options for ethical, legal, and economic solutions to age-related problems. Agequake, named after the phenomenon of the rapidly growing number of older people in prison, is one of the few research projects that provides data on the older prison population and contributes to the knowledge of this underresearched group. Through capturing the reality concerning mental health of older incarcerated adults, the findings provide an original insight into the prevalence of mental health problems, types of treatment provided and access to mental health care. Furthermore, strategies are provided to improve the physical and mental health care and reduce the burden on prison staff and the public at large.
\end{abstract}

Keywords Ageing · Elder · Prison - Imprisoned · Psychiatric · Therapy

Die Autorinnen Helene Seaward und Laudelina Taboas Hidalgo haben zu gleichen Teilen zum Manuskript beigetragen.

\footnotetext{
$\triangle$ Dr. Helene Seaward

helene.seaward@unibas.ch

Laudelina Taboas Hidalgo
laudelina.taboashidalgo@unibas.ch

Laudelina Taboas Hidalgo
laudelina.taboashidalgo@unibas.ch

1 Institut für Bio- und Medizinethik, Universität Basel, Basel, Schweiz

2 L'institut universitaires de médecine légale, Faculté de médecine, L’Université de Genève, Genf, Schweiz

médecine, L'Université de Genève, Genf, Schweiz
}

\section{Einführung}

In diesem Übersichtsartikel werden die bisherigen Ergebnisse verschiedenster Studien innerhalb des Forschungsprojekts Agequake II in Kurzform aufgezeigt. Um die Ergebnisse möglichst übersichtlich darstellen zu können, werden die einzelnen Studien also ohne Anspruch auf Vollständigkeit in komprimierter Form berichtet. Die zitierfähigen Ergebnisse sind in den Originalarbeiten der Studien ersichtlich. 


\section{Hintergrund}

Das Phänomen der rapide wachsenden Anzahl älterer inhaftierter Personen (Walmsley 2013), ,agequake“, und die damit verbundenen Veränderungen stellen die Gesundheitsversorgung in Gefängnissen vor neue Herausforderungen (Trotter und Baidawi 2015). Da die Datenlage zur psychischen Gesundheit inhaftierter Personen spärlich ist, bedarf es zur Erarbeitung von Lösungsansätzen dringlich eingehender Forschung.

Die Zahl der inhaftierten Personen nimmt weltweit zu (Walmsley 2016), während ältere Menschen die am schnellsten wachsende Altersgruppe in Justizvollzugsanstalten sind (Baidawi und Trotter 2016; Di Lorito et al. 2018). In der Schweiz stieg der Anteil der inhaftierten Erwachsenen im Alter über 49 Jahre im Straf- und Maßnahmenvollzug von 6,6\% im Jahr 1984 auf 17,7\% im Jahr 2019 an (Bundesamt für Statistik 2020b). Wenn man den Maßnahmenvollzug separat betrachtet, so fällt diese Entwicklung noch extremer aus, da Personen im Alter über 49 Jahre im Jahr $19848 \%$ aller zu einer Maßnahme verurteilten Personen ausmachten; im Jahr 2019 ist deren Anteil auf 28\% angestiegen (Bundesamt für Statistik 2020a). Im schweizerischen Justizvollzug ist die steigende Zahl älterer Inhaftierter daher v. a. bei den Behandlungspflichtigen zu beobachten. Da fast jede dritte Person über 49 Jahre alt ist, stellen sie keine Minderheit mehr dar, sondern sind eine häufige Gruppe von PatientInnen. Der Grund für dieses exponentielle Wachstum der älteren Bevölkerung in Justizvollzugsanstalten ist auf verschiedene Entwicklungen zurückzuführen (Leigey und Hodge 2012; Yarnell et al. 2017). Ihr Wachstum kann teilweise auf die Alterung der Gesellschaft zurückgeführt werden, die sich in der Gefängnispopulation widerspiegelt. Steigende Kriminalitätsraten bei älteren Menschen tragen zum Anstieg der älteren Bevölkerung in Justizvollzugsanstalten bei (Fazel und Baillargeon 2011; Sodhi-Berry et al. 2015). Auch wenn ein Teil dieses Anstiegs auf die allgemeine Alterung der Gesellschaft zurückzuführen ist, so ist doch der größte Teil auf eine härtere Strafverfolgungspolitik und strengere Strafvollzugspraktiken zurückzuführen. Längere Haftstrafen und eine restriktive Bewährungspolitik tragen daher dazu bei, dass eine größere Zahl von Personen im Gefängnis altert (Fazel und Baillargeon 2011; Jang und Canada 2014; Turner und Peacock 2017). Die Zahl an älteren inhaftierten Personen nimmt nicht nur mit einem rasanten Tempo zu, sie sind zusätzlich die Altersgruppe mit den größten gesundheitlichen Bedürfnissen. Ältere Insassen haben die höchsten Prävalenzraten von somatischen und psychischen Erkrankungen im Vergleich zu jüngeren Insassen als auch im Vergleich Personen im gleichen Alter in der Allgemeinbevölkerung (Fazel et al. 2001; Wangmo et al. 2015). Sie nehmen daher im Verhältnis zu ihren absoluten Zahlen relativ einen deutlich größeren Anteil der Gesundheitsversorgung in Anspruch. Aus diesem Grund wird auch vermutet, dass die ältere Gefängnispopulation einen wesentlichen Faktor in den steigenden Kosten der Gefängniskosten spielen (Al-Rousan et al. 2017). Aufgrund der hohen gesundheitlichen Bedürfnisse der älteren Insassen ist es daher besonders wichtig, deren Bedürfnisse detailliert zu beleuchten sowie Interventionsstrategien $\mathrm{zu}$ optimieren (Baidawi und Trotter 2015). Dies nicht nur um den individuellen Bedürfnissen des Einzelnen besser gerecht zu werden, sondern auch, um eng begrenzte Ressourcen im Gefängniskontext bestmöglich zu nutzen sowie den steigenden Kosten entgegenzuwirken. Dies um den Gesundheitszustand des Einzelnen zu fördern, aber auch, um die Anpassungsleistung der älteren Insassen an die Gefängnisumgebung zu fördern sowie deren Rückfallrisiko zu minimieren.

Weltweit fehlen systematische Daten zur Gesundheit alternder Menschen im Gefängnis sowie deren Gesundheitsversorgung. Bisherige Studien weisen jedoch darauf hin, dass über $80 \%$ der älteren Personen an einer chronischen Erkrankung leiden (Fazel et al. 2001). Spezifisch in Bezug auf die psychische Gesundheit, leiden laut WHO bis zu $40 \%$ der GefängnisinsassInnen an psychischen Problemen (World Health Organization 2015) und $10-15 \%$ an dauerhaften psychischen Erkrankungen wie beispielsweise Schizophrenie, bipolaren Störungen oder Autismusstörungen (World Health Organisation 2014). Unter den älteren inhaftieren Personen leidet schätzungsweise jede zweite Person an einer psychiatrischen Erkrankungen (Kakoullis et al. 2010; Kingston et al. 2011). In der Schweiz gibt es jedoch keine systematische Datenlage zur psychischen Gesundheit alternder Gefangener. Unsere vom SNF geförderten Forschungsprojekte „Agequake I und II“ erhoben daher Daten rund um die somatische und psychische Gesundheit von älteren inhaftierten Personen sowie deren Gesundheitsversorgung in Schweizer Gefängnissen. Agequake I konzentrierte sich auf somatische Erkrankungen (Elger et al. 2015; Wangmo et al. 2014). Durch die Analyse der Gesundheitskosten innerhalb des Gefängnisses in Bezug auf verschiedene Krankheitsbilder in verschiedenen Alterskohorten und Geschlechtergruppen konnten so Charakteristiken gefunden werden, die zu kennen zu einem effizienteren Umgang medizinischer Ressourcen im Gefängnis führen kann (Moschetti et al. 2015, 2018). Agequake I hat weiterhin wertvolle Informationen zu den Bedürfnissen bezüglich der Wohnsituation, der Ernährung im Gefängnis und Problemen mit der Unterbringung älterer inhaftierter Personen geliefert (Wangmo et al. 2014; Wangmo et al. 2016) und ist ethische Fragestellungen zu Tod, Sterben und Entscheidungen des Lebensendes angegangen (Handtke et al. 2017; Wangmo et al. 2017). So konnten die Faktoren identifiziert werden, die eine bedingte Entlassung erschweren. Außer- 
dem wurden die ethischen Gesichtspunkte zu Bedürfnissen bezüglich des Lebensendes und deren Implementierung, die im Gefängnis vonnöten wäre, hervorgehoben.

Aus Agequake I ging zudem hervor, dass die medizinischen Aufzeichnungen in den Gefängnissen je nach Gefängniskontext und Organisation stark variieren. Da die Agequake-I-Daten aus den Krankenakten der Gefängnisse erhoben wurden, war es für viele Gefängnisse nicht möglich, Daten aus den psychiatrischen Akten ebenfalls einzubeziehen. Gefangene zögern zudem oftmals, psychische Probleme aus Angst vor Stigmatisierung (Howerton et al. 2007) offenzulegen, weshalb eine mangelhafte Diagnostizierung durch Mitarbeitende ohne Fachwissen wahrscheinlich ist. Darüber hinaus priorisieren in Gefängnissen angestellte HausärztInnen und Pflegepersonal dringende somatische Beschwerden, da sie einer hohen Arbeitsbelastung ausgesetzt sind: Das Europäische Komitee zur Verhütung von Folter (CPT) hat kürzlich empfohlen, die Präsenz von medizinischem Personal in Schweizer Gefängnissen zu erhöhen (Council of Europe: Committee for the Prevention of Torture 2012). Aus diesen Ergebnissen zur allgemeinen Gesundheitsversorgung resultierte die Ausführung des Folgeprojekts Agequake II, das wichtige neue Forschungsdaten zur psychischen Gesundheit alternder Gefangener erhob.

Die psychiatrische Gesundheitsversorgung von inhaftierten Personen findet in einem komplexen Kontext inmitten der Interaktionen zwischen medizinischen und juristischen Institutionen statt. Es ist dabei sinnvoll, zwei Arten von psychischen Gesundheitsinterventionen für Gefangene zu unterscheiden. Der erste Typ umfasst therapeutische Interventionen für Gefangene mit einer Freiheitsstrafe. In diesem Fall gelten im Gefängnis alle ethischen und rechtlichen Bedingungen des ÄrztInnen-PatientInnen-Verhältnisses, wodurch eine Gleichwertigkeit der Behandlung wie bei PatientInnen außerhalb von Gefängnissen gewährt werden soll (Elger 2008; SAMW 2002). In dem häufig bestehenden Gatekeeper-System werden Gefangene aber nur dann an psychosoziales Personal überwiesen, wenn sie sich spontan beschweren und die HausärztInnen das Problem für ausreichend schwerwiegend halten (Bretschneider und Elger 2014).

Die zweite Art von Interventionen im Bereich der psychischen Gesundheit betrifft gerichtlich angeordnete Therapien für Gefangene, die zu therapeutischen Maßnahmen verurteilt wurden. PsychiaterInnen sehen sich mit einer ethisch schwierigen Doppelrolle als Therapierende konfrontiert, die zusätzlich die Verpflichtung haben, der Justiz über den Behandlungsverlauf zu berichten (Elger et al. 2015; Wangmo et al. 2014). Die Gruppe der PatientInnen, welche zu einer Maßnahme verurteilt wurden, nimmt weltweit und auch in der Schweiz deutlich zu (Riklin 2014) und bildete 2013 zusammen mit sehr langen Freiheitsstrafen $(\geq 10$ Jahre und lebenslänglich) $13 \%$ aller verurteilten Gefangenen in Euro- pa (Aebi und Delgrande 2013). Agequake I bestätigte, dass der Anstieg der alternden Gefangenen durch die massive Zunahme von Personen, welche sich in einer Maßnahme nach Artikel 59 (stationäre Maßnahme) oder 64 (Verwahrung) StGB angetrieben wird, wobei Art. 59 eine Behandlung unter der Aufsicht von forensischen Psychiatern und Psychologen vorschreibt.

Gegenwärtig gibt es international wenige systematische Datenerhebung über die therapeutischen Interventionen für alternde Häftlinge. Das Fehlen dieser Informationen ist u. a. auf die variablen innerstaatlichen Gesetze und länderspezifischen, oft föderal organisierten Verfahrensordnungen zurückzuführen. Die daraus resultierende Spaltung ist sowohl für den Bereich der forensischen Psychiatrie als auch für die Gesetzgebung und öffentliche Politik nachteilig. Eine weitere Tätigkeit, die von psychiatrischem Personal im Rahmen dieser Maßnahmen durchgeführt wird, ist die Beurteilung des zukünftigen Risikos, sprich der „Gefährlichkeit“. Diese Bewertungen bilden die Grundlage für Entscheidungen der Justiz, eine Maßnahme aufrechtzuerhalten oder eine Entlassung zu gewähren. Obwohl die endgültige Entscheidung über die Verhängung, Verlängerung oder Aufhebung von Sicherheitsmaßnahmen in den meisten Ländern von der Justiz getroffen wird, ist die forensische Einschätzung des Rückfallrisikos von entscheidender Bedeutung. Der Bereich der Risikobewertung hat sich in den letzten Jahren erheblich weiterentwickelt und versucht, Erkenntnisse aus strukturierten Instrumenten wie HCR-20 (Habermeyer 2009) und PCL-R (Mokros et al. 2011) sowie aktuarischen Instrumenten wie VRAG, SORAG, Static 99 (Rossegger et al. 2013) und SVR-20 (Dietiker et al. 2007) zu integrieren. Dennoch gibt es eine Reihe von methodischen Schwierigkeiten aufgrund von Referenzstichproben, Untergruppenund Gewalttypenvariationen sowie der Tatsache, dass die meisten Instrumente nur selten unter europäischen Gefängnispopulationen validiert wurden (Mokros 2015). Weltweit und in der Schweiz bleibt die Vorhersage der Rückfälligkeit für einzelne StraftäterInnen wissenschaftlich problematisch (Glancy 2006; Mercado und Ogloff 2007; Mokros 2015; Rossegger et al. 2013). Die Tatsache, dass verschiedene ExpertInnen unterschiedliche Kombinationen von Tests verwenden, erschwert die Erhebung systematischer Daten zusätzlich (Dittmann 2003; Habermeyer et al. 2008, 2009).

In ähnlicher Weise ist es aufgrund variabler Behandlungsmethoden und -programme schwierig, Anhaltspunkte für die Effektivität forensischer Behandlungen zu erhalten, beispielweise in Bezug auf die soziale Funktionsfähigkeit und das Risiko gewalttätigen Verhaltens (Långström et al. 2013; Trestman et al. 2007). Weltweit, und dies gilt auch für viele Schweizer Kantone, fehlt es an universitär angeschlossener oder zumindest akademisch-partnerschaftlicher psychiatrischer Versorgung in Gefängnissen (R. L. Trestman et al. 2015; Wakai et al. 2009). Solche universitären 
Zentren haben sich als sehr wichtig für die Entwicklung und wissenschaftliche Beurteilung neuer Behandlungen erwiesen (Kersten et al. 2016).

Die Folgen fehlender oder inadäquater Behandlung können zudem gravierend sein: Angstzustände, Depressionen und Substanzmissbrauch (Elger 2004; Elger et al. 2002; R. Trestman et al. 2015) erhöhen signifikant das Suizidrisiko im Gefängnis. Suizid stellt eine führende (und vermeidbare) Todesursache in Gefängnissen der westlichen Hemisphäre dar (Ruiz et al. 2014; Spellman und Heyne 1989; Wangmo et al. 2014). Obwohl ältere Personen in Schweizer Gefängnissen immer noch eine Minderheit darstellen, stellen sie besonders hohe Anforderungen an die forensischpsychiatrische Versorgung, was spezifische Strategien zur Behandlung dieser Gruppe erfordert.

\section{Methodik}

Wir erhoben daher Daten zur psychischen Gesundheit älterer inhaftierter Personen, evaluierten die aktuelle Situation deren psychiatrischer Versorgung und untersuchten Optionen $\mathrm{zu}$ ethischen, rechtlichen und wirtschaftlichen Lösungen in Bezug auf altersbedingte Probleme. Die nationalen und internationalen Richtlinien werden als Garantie für angemessene Gesundheitsversorgung älterer Gefangener ausgelegt, die Details zur Durchsetzbarkeit dieser Gesetze sind jedoch nicht geregelt und daher die benötigte medizinische Versorgung nicht garantiert. Die rechtliche Situation unterstreicht die Notwendigkeit einer soliden ethischen Argumentation, um alternde Inhaftierte schützen zu können. Durch die Untersuchung der aktuellen Situation älterer inhaftierter Personen mit psychischen Gesundheitsproblemen und jenen, die eine Sicherheitsmaßnahme verbüßen, zielt die Studie auf eine Verbesserung des medizinischen, sozialen und rechtlichen Status und der Lebensqualität dieser Gruppe von Gefangenen ab.

Der für die Studie verwendete ,Mixed-methods“-Ansatz umfasste qualitative und quantitative Datenerhebungen (Tab. 1). Mittels systematischen Übersichtsarbeiten identi-

Tab. 1 Stichprobenmerkmale

\begin{tabular}{ll}
\hline Qualitative Datenerfassung & \\
\hline PatientInnen: & Total $n=50$ \\
Inhaftierte Erwachsene im Alter & $n=41$ zu Maßnahmen (gemäß \\
von 50 Jahren und älter, die eine & Art. 59, 63 und 64 des StGB) \\
psychiatrische und psychothera- & verurteilte Personen \\
peutische Behandlung erhalten & zu Freiheitsstrafen verur- \\
& teilte Personen \\
ExpertInnen: & $\begin{array}{l}\text { Total } n=73 \\
n=29 \text { aus der Schweiz } \\
\text { Fachkräfte in der Psychiatrie und } \\
\text { Psychologie, die ältere Inhaftier- } \\
\text { te behandeln (PsychiaterInnen, }\end{array}$ \\
$\begin{array}{l}\text { PsychologInnen, psychiatrische } \\
\text { Pflegekräfte) }\end{array}$ & \\
\hline
\end{tabular}

fizierten wir zuerst die aktuelle Literatur zur psychischen Gesundheit dieser Population. Dies mit einem spezifischen Fokus auf die Prävalenzraten von psychiatrischen Erkrankungen der älteren Gefängnispopulation, v.a. im Vergleich mit jüngeren Inhaftierten. Weiterhin identifizierten wir zentrale Hürden in der Integration der Literatur und legten einen spezifischen Fokus auf die fehlende Definition der höheren Altersgruppe, was die Zusammenführung der begrenzten Literatur erschwert.

Einen zentralen Teil unsere Forschungsarbeit widmeten wir der qualitativen Datenerhebung. Wir interviewten ExpertInnen im Bereich von Strafvollzug und forensischer Psychiatrie zur psychischen Gesundheitsversorgung und zur Beurteilung der Legalprognose von StraftäterInnen. Dabei strebte die Studie die Erfassung von verschiedenen Strategien innerhalb des Gesundheitssystems und Meinungen zu deren Umsetzung im Gefängnis an. Ein weiteres Ziel des Projekts war, ExpertInnen aus Kanada zu befragen, um andere Strategien in der psychischen Gesundheitsversorgung zu verstehen und ihre mögliche Umsetzung im Schweizer System einschätzen zu können. So sollten Unterschiede von Vorteilen und Schwächen verschiedener Ansätze zur psychischen Gesundheit mit besonderem Fokus auf die Behandlung von alternden PatientInnen im Maßnahmenvollzug identifiziert werden.

Zusätzlich wurden ältere PatientInnen zu ihrer Sichtweise der psychischen Gesundheitsversorgung und zu ihrem Leben mit einem psychischen Leiden im Straf- und Maßnahmenvollzug befragt. Somit konnten Sichtweisen von inhaftierten Personen und ExpertInnen zur psychischen Gesundheitsversorgung und der Risikoeinschätzung erforscht werden.

Es wurden halbstrukturierte Interviews mit inhaftierten Personen im Alter von über 50 Jahren und ExpertInnen für psychische Gesundheit im Gefängniskontext geführt. Dabei handelte es sich um PsychologInnen, PsychiaterInnem, psychiatrisches Pflegepersonal, SozialarbeiterInnen und ErgotherapeutInnen. Eingeschlossen wurden forensischpsychiatrische Einrichtungen und Justizvollzugsanstalten in der Schweiz und in Kanada, wobei die Interviewsprachen Deutsch, Schweizerdeutsch, Französisch und Englisch waren. Ausgeschlossen wurden Einrichtungen, in denen jugendliche Personen untergebracht sind, oder Einrichtungen, die im Zusammenhang mit der Ausschaffung von Asylsuchenden stehen, sowie italienischsprachige Regionen der Schweiz. Alle inhaftierten Personen wurden über eine Kontaktperson der psychosozialen Dienste oder der Gefängnisverwaltung kontaktiert. Die befragten Personen erhielten keine Entschädigung für ihre Teilnahme. Zwei Mitglieder des Forschungsteams wurden in der qualitativen Datenerhebung geschult und während des gesamten Prozesses der Datenerhebung beaufsichtigt. Der Interviewleitfaden für inhaftierte Teilnehmende ermittelte Informationen über 
(a) persönliche Umstände und soziale Netzwerke, (b) Erfahrungen mit dem Älterwerden im Gefängniskontext, (c) Zugang zu und Qualität der psychischen Gesundheitsversorgung, (d) Zufriedenheit mit der psychischen Gesundheitsversorgung, (e) psychisches Wohlbefinden und (f) Erfahrungen mit Risikobeurteilungen.

Die teilnehmenden ExpertInnen wurden gebeten, ihre Sichtweise $\mathrm{zu}$ folgenden Themen darzulegen: (a) Motivation, mit inhaftierten Personen zu arbeiten, kurze Beschreibung ihrer Verantwortlichkeiten, (b) Organisation der psychosozialen Versorgung, Meinung über den Zugang zu und die Qualität von psychosozialen Versorgungsdiensten, Einfluss von unbefristeten Entlassungsdaten auf die Arbeit mit ihren PatientInnen, (c) Untersuchung ihrer Erfahrungen in der Arbeit mit älteren PatientInnen, ihre Meinung über Merkmale der Versorgung und Interaktion mit älteren PatientInnen, Ähnlichkeiten und Unterschiede in der Betreuung jüngerer und älterer PatientInnen, prominente Therapiethemen bei älteren PatientInnen, (d) Doppelrollenkonflikt der Therapierenden, Beschreibung der Zusammenarbeit mit anderen Berufsgruppen und Vertretenden der Justiz, und (e) Risikobewertung und Meldung an die Behörden: Merkmale, Verfahren, Alter als Variable in der Risikobewertung, Schlüsselkriterien in den Meldestandards.

Die Analyse der erhobenen Daten folgte dem Konzept der thematischen Analyse nach Braun and Clarke (2006). Mit dem Softwareprogramm MAXQDA wurden die Interviews transkribiert und im Anschluss erste Codes erstellt. Um einen einheitlichen Kodierungsbaum für das gesamte Projekt sicherzustellen, wurden zunächst 8 Interviews vom Studienteam gelesen und kodiert. Mittels Diskussion der in den Daten sichtbaren Nuancen legte das Studienteam die verschiedenen Codes fest. Die weiteren Transkripte wurden von einzelnen Teammitgliedern kodiert und die neuen Codes im Plenum besprochen, um hierdurch den finalen Kodierungsbaum zu bestimmen.

\section{Studienergebnisse}

\section{Prävalenzraten und die Notwendigkeit einer einheitlichen Altersgrenze für ältere inhaftierte Personen}

Die systematischen Übersichtsarbeiten ermöglichten es uns, Prävalenzraten psychiatrischer Störungen speziell für ältere inhaftierte Erwachsene zu skizzieren (Haesen et al. 2018). Die Untersuchung der Prävalenz psychischer Erkrankungen in der Gruppe der älteren Strafgefangenen bestätigte den Mangel an zuverlässigen Daten (Haesen et al. 2018). Trotz bestehender Einschränkungen deutet die Literatur tendenziell auf eine höhere Prävalenz psychischer Störungen bei älteren Gefangenen im Vergleich zu jüngeren Gefangene sowie gleichaltrigen Personen der Allgemeinbevölkerung hin. Im Besonderen leiden ältere Gefangene häufiger an Alkoholabhängigkeit, affektiven Störungen sowie kognitiven Einschränkungen (Davoren et al. 2015; M. L. Gates et al. 2017; Lewis et al. 2006; Sodhi-Berry et al. 2015). Außerdem sind neurodegenerative Störungen wie z. B. Demenz, die für die alternde Bevölkerung typisch sind, in diesen Studien nur teilweise berücksichtigt und erfordern spezifische Ansätze. Eine detaillierte Übersicht der Prävalenzzahlen ist in der Originalarbeit von Haesen et al. (2018) ersichtlich.

Weiterhin analysierten wir die Art der Definition der älteren Altersgruppe, um ein gemeinsames Verständnis für die Altersgruppe der älteren Inhaftierten zu entwickeln (Merkt et al. 2020). Dies ist besonders wichtig, da ältere inhaftierte Erwachsene derzeit mit unterschiedlichen chronologischen Altersgrenzen zwischen 40 und 65 Jahren definiert werden, was die Integration der ohnehin sehr begrenzten Forschung erschwert. Wir haben daher nicht nur den aktuellen Stand der Forschung zur psychischen Belastung älterer inhaftierter Erwachsener skizziert, sondern auch die aktuellen Mängel der vorhandenen Literatur in diesem Bereich aufgezeigt.

Weiterhin deuten unsere Ergebnisse darauf hin, dass die Anwendung des Alters 50 zur Definition der älteren Gefängnispopulation derzeit am besten geeignet ist. Dies, weil Konzepte wie das vorzeitige Altern aufgrund der unvorteilhaften Lebensbedingungen vor und während der Inhaftierung die Morbidität und Mortalität beeinflussen. Dies resultiert in einen drastischen Anstieg der Prävalenzraten von psychischen Krankheiten und den damit verbundenen Behandlungskosten ab Alter 50, was für eine Trennung der jüngeren und älteren Personen in Bezug auf die Versorgungsplanung mit Alter 50 spricht. Diese Konzepte sind vielversprechende Ansätze, die es weiter zu verfolgen gilt, um ein gemeinsames Verständnis älterer Menschen im Gefängnis zu fördern und einheitlich zu erforschen. Wir regen jedoch an, ggf. Untersuchungen mit allen Altersgruppen durchzuführen, um Veränderungen zwischen den Kohorten zu erkennen, damit die Zuteilung der begrenzten Ressourcen im Gesundheitswesen angemessen geplant werden kann.

\section{Die spezifischen Herausforderungen einer gerichtlich angeordneten Therapie für ältere inhaftierte Personen}

Unsere Untersuchungen zeigen wichtige Erkenntnisse zur Behandlung älterer PatientInnen, die per Gerichtsbeschluss zu einer Therapie verpflichtet wurden. Solche Behandlungssettings sind aufgrund individueller Faktoren wie der Schwere der psychischen Probleme und der Häufigkeit von Komorbidität sowie des Zwangscharakters der Gefängnisumgebung mit besonderen Herausforderungen verbunden. 
Darüber hinaus spielt die Justizbehörde eine wichtige Rolle, da sie die Festlegung der Behandlungsbedingungen, -themen und -ziele beeinflusst. Diese Einflüsse sind gleichermaßen entscheidend für den Therapieprozess, für die therapierenden Fachkräfte sowie für die PatientInnen und haben das Potenzial, die Effektivität der gerichtlich angeordneten Interventionen sowohl in Bezug auf strafrechtliche als auch psychiatrische Ergebnismessungen zu beeinflussen (Seaward et al. 2021).

Unsere Ergebnisse deuten darauf hin, dass psychosoziale Fachkräfte mit der Integration ihrer Doppelrolle von Betreuung und Kontrolle zu kämpfen haben, insbesondere im Hinblick auf fehlende normative und praktische Richtlinien, die die klinische Arbeit leiten. Ihre individuellen Strategien im Umgang mit diesen Herausforderungen lassen sich dahingehend zusammenfassen, dass sie Wege finden, eine vertrauensvolle Begegnung zu fördern, indem sie die PatientInnen transparent über die Behandlungsbedingungen und die Erwartungen der Justizbehörden informieren (Merkt et al. 2020). Aktuelle Grenzen der Vermittlung von Transparenz sind die fehlende Übereinstimmung zwischen PatientIn, TherapeutIn und Justizbehörden über Behandlungsziele und damit verbundene Konsequenzen für Vollzugslockerungen und Entlassungstermine. Weiterhin müssten aus ethischen und behandlungsbezogenen Gründen die beiden Rollen der Betreuung und Kontrolle von zwei verschiedenen Personen wahrgenommen werden, deren Verantwortlichkeiten klar definiert sind. Beispielsweise wurde die eingeschränkte Schweigepflicht von den Teilnehmenden akzeptiert, vorausgesetzt, dass die Weitergabe von Informationen transparent und klar kommuniziert wurde. Jedoch scheint eine klare Trennung zur entscheidenden Instanz vorteilhaft zu sein, insbesondere bezüglich der Kommunikation von Aspekten wie z. B. Vollzugslockerungen. Dies insbesondere, um es den Therapierenden zu ermöglichen, ausschließlich eine betreuende Rolle einzunehmen, um so eine qualitativ hochwertige therapeutische Allianz zu fördern, die letztlich verbesserte Behandlungsergebnisse ermöglicht.

Darüber hinaus beeinflusst die Art und Weise, wie externer Druck durch die Justizbehörden ausgeübt wird, die Motivation der PatientInnen. TherapeutInnen spielen eine entscheidende Rolle, indem sie die Forderungen der Behörden an die PatientInnen vermitteln und gleichzeitig versuchen, die Motivation der PatientInnen zur Teilnahme an der Therapie aufrechtzuerhalten. Insgesamt haben unsere Ergebnisse gezeigt, dass TherapeutInnen derzeit bei der Suche nach Strategien zum Umgang mit den besonderen Herausforderungen bei gerichtlich angeordneten Behandlungen allein gelassen werden. Beispielsweise wird der Umgang mit der eingeschränkten Schweigepflicht und der daraus resultierenden Doppelrolle der TherapeutInnen aktuell auf unterschiedliches Art und Weise geregelt. Obwohl überge- ordnet eine transparente Kommunikation als Strategie zum Umgang mit dem Kontakt zum Justizsystem von der Mehrheit aufgeführt wird, bleiben die tatsächlichen Strategien im klinischen Alltag divers. Aufgrund der fehlenden Richtlinien zum Umgang mit der Doppelrolle sind TherapeutInnen daher gezwungen, individuelle Lösungsansätze zu finden, ohne die konkrete Auswirkung auf den Prozess der psychotherapeutischen Behandlung zu kennen.

Weiterhin betonten Experten, dass die Therapierenden einen zusätzlichen Fokus darauf legen sollten, eine respektvolle, wertschätzende und positive Haltung zu zeigen. Diese Strategie könnte der Angst von Gefangenen, aufgrund ihres Status diskriminiert zu werden, entgegenwirken. Inhaftierte Personen sind oft etikettierenden Haltungen ausgesetzt, was sich negativ auf das eigene Selbstwertgefühl auswirkt. Als Therapierende eine respektvolle Haltung einzunehmen, ist also auch deshalb wichtig, weil durch ein geringes Selbstwertgefühl Veränderungen potenziell behindert werden. Weiter zeigten die Ergebnisse, dass es von besonderer Bedeutung ist, den PatientInnen ein gewisses Maß an Wahlmöglichkeiten und Kontrolle über den Inhalt und den Verlauf der Therapie einzuräumen. PatientInnen fühlen sich durch die Möglichkeit, ihre persönlichen Anliegen einzubringen, respektiert und wertgeschätzt. Die PatientInnen betonten darüber hinaus, dass es von zentraler Bedeutung ist, sich ausführlich mit den eigenen Problemen zu befassen und die Behandlung mit sehr spezifischen und konkreten Beispielen aus der aktuellen Lebenssituation anzugehen. So würde das Verständnis des begangenen Delikts gestärkt. Dadurch werden die PatientInnen zur aktiven Teilnahme an der Behandlung motiviert. Feedback und Ratschläge sollten sich folglich auf die aktuelle Situation der Person beziehen, sowie konkret und detailliert sein, vage und allgemeine Kommentare also vermieden werden.

Der Prozess forensisch-psychiatrischer Risikobeurteilung wurde von Wangmo et al. (2021) genauer beleuchtet. Die AutorInnen machen auf den Bedarf aufmerksam, dass der Ablauf forensisch-psychiatrischer Risikobewertungen und die darauf basierenden Entscheidungen, klarer kommuniziert werden sollten. Im Besonderen sollte sich die inhaftierte Person die konkreten Ziele und Anforderungen der Vollzugsbehörden im Hinblick auf deren Behandlungsziele kennen. Aus diesem Grund sollten Therapieziele zwischen allen Beteiligten detailliert abgestimmt sein, damit der Patient den Eindruck erhält, dass die Entscheidungen der Vollzugsbehörden objektiv gefällt werden und gerechtfertigt sind. Eine solche Transparenz und Klarheit ist nicht nur im Hinblick auf Motivation und Behandlungszufriedenheit der betroffenen Personen wichtig, sondern auch für das Justizsystem in Bezug auf den Effekt der verordneten Therapie auf kriminelle Rückfallraten. 


\section{Haftbedingungen und deren Auswirkungen auf die psychische Gesundheit}

Wir konnten als eine der ersten Studien die Perspektive inhaftierter Personen auf Einsamkeit, soziale Isolation und fehlende soziale Kontakte in Schweizer Gefängnissen aufzeigen (Felix Pageau et al., under review). Vor allem die eingeschränkte Bewegungsfreiheit und fehlendes soziales Kapital in Form von Vertrauen sowie der begrenzte Kontakt zum Gefängnispersonal sind entscheidende Hindernisse für die Knüpfung sozialer Beziehungen. Da soziale Beziehungen wichtige Elemente für das psychische Wohlbefinden sind, ist es wichtig, Möglichkeiten für Gefangene zu schaffen, Beziehungen aufzubauen und zu pflegen. Dies ist insbesondere für die ältere Altersgruppe entscheidend, die häufig eine langfristige Inhaftierung erlebt. Um diese Isolation zu verringern, ist es außerdem wesentlich, auch das Gefängnispersonal zu einem vertrauensvollen Beziehungsaufbau zu den inhaftierten Personen zu schulen.

Psychische Krankheiten begünstigen eine Abwärtsspirale zwischen ungesundem Lebensstil und schlechter psychischer Gesundheit: unbehandelte psychische Erkrankungen wirken sich negativ auf die Ernährung, körperliche Aktivität und den Drogenkonsum aus (Félix Pageau et al., under review). Unseren Befragungen zufolge haben ältere Gefangene oft keinen Zugang zu Lebensmitteln, die an ihre gesundheitlichen Probleme angepasst sind. Dies wurde am Beispiel von sehr fettigem, cholesterinhaltigem Essen, das für Gefangene mit Herzproblemen besonders schädlich sein kann, beschrieben. Das Sportangebot ist meist auf jüngere Personen zugeschnitten und nicht für ältere Gefangene geeignet. Des Weiteren behindern Langeweile und mangelnde Verantwortung einen Wandel hin zu einem besseren Lebensstil für ältere Erwachsene im Gefängnis. Die Ergebnisse zeigen auf, dass eine Änderung der Gefängnisumgebung sich indirekt über einen Wandel der Lebensgewohnheiten auf die psychische Gesundheit auswirken kann.

\section{Zusammenfassung der Ergebnisse}

Aus den einzelnen Studienergebnissen zeigt sich, dass die psychische Gesundheit älterer Gefangener von besonderer Relevanz für die Forschung, Ressourcenplanung und Umsetzung von psychischer Gesundheitsversorgung im Gefängnis ist. Gefangene ab 50 Jahre, die häufig von vorzeitigem Altern betroffen sind, zeigen eine höhere Prävalenz von psychischen Erkrankungen auf. Das bedingt einen hohen Behandlungsbedarf dieser Population und kann zu höheren Behandlungskosten dieser Altersgruppe beitragen.

Im Kontext von gerichtlich angeordneter Therapie zeigen unsere Ergebnisse, dass eine transparente Kommunikation sowohl vonseiten der Therapierenden als auch von der Justizbehörde von großer Bedeutung ist. Diese Transparenz wird jedoch durch nichtübereinstimmende Behandlungsziele von PatientInnen, TherapeutInnen und Justizbehörde behindert. Dass sich Behandlungsziele unterscheiden, wirkt sich für alle drei Parteien negativ auf eine gelungene Therapie aus. Es ist daher wichtig, die Therapieziele detailliert in Kommunikation mit allen Parteien aufeinander abzustimmen. So können die PatientInnen den Entscheidungen der Vollzugsbehörden mehr Verständnis entgegenbringen und diese als gerechtfertigt ansehen. In Bezug auf die funktionierende therapeutische Beziehung und die Therapiemotivation der PatientInnen wäre es wichtig, wertschätzend auf die PatientInnen zuzugehen. Den PatientInnen eine gewisse Wahlmöglichkeit für den Therapieverlauf und deren Inhalt zu geben, zeigte sich weiter als eine gute Strategie für klare Behandlungsziele und erhöhte Therapiemotivation.

Letztlich zeigten die qualitativen Daten, dass sich die Haftbedingungen gerade für ältere Menschen negativ auf die psychische Gesundheit auswirken können, beispielsweise weil der Aufbau sozialer Beziehungen im Gefängnis deutlich erschwert ist. Eine Anpassung der Gefängnisumgebung an die Bedürfnisse älterer Menschen könnte durch gesünderes Essen und das Ermöglichen sozialen Beziehungsaufbaus positiv Einfluss auf einen gesünderen Lebensstil und damit auf die psychische Gesundheit nehmen.

Zusammenfassend lässt sich sagen, dass wir mit einer Population geforscht haben, die häufig vergessen wird und schwer zugänglich ist. Unsere Forschung trägt daher zur Erweiterung des Wissens über diese sehr wenig erforschte Gruppe bei und stellt mögliche Strategien zur Überwindung der aktuellen Herausforderungen bei der Unterbringung und Behandlung älterer inhaftierter Erwachsener vor. Unsere Arbeit trägt auch dazu bei, die Wahrnehmungen älterer inhaftierter Erwachsener besser zu verstehen und so vulnerable Personen durch Verbesserungen organisatorischer Aspekte des Gefängnislebens vor Schaden zu schützen. Dies ist nicht nur wichtig, um die Achtung der PatientInnenrechte und der Menschenrechte i. Allg. zu gewährleisten, sondern auch, um die kriminelle und psychiatrische Rückfälligkeit der einzelnen inhaftierten Person zu reduzieren, um letztlich die Belastung des Gefängnispersonals und gleichzeitig der größeren Öffentlichkeit zu verringern. Die Ergebnisse des Projekts sind daher von hohem Interesse für ÄrztInnen, EthikerInnen, ÖkonomInnen und SoziologInnen, für politische Entscheidungstragende, Gefängnisverwaltende, NGO und internationale Organisationen.

Danksagung Wir danken unseren Studierenden für die Transkription der Interviews: Antonina Brunner, Chiara Andenmatten, Déborah Schorno, Emely Schweizer, Flavienne Bieri, Laryssa Grosjean, Laudelina Taboas Hidalgo, Leila Meyer, Luisa Waschkowski, Sabrina Wenger, Sasa Pospisilova, Sophie Dieffenbacher, Valentina Memma, Vivianne Götz, Bianca Ballaman, Ziad Kassem, Yael Becker. Wir danken allen Fachleuten aus dem Bereich der psychischen Gesundheit und den inhaftierten Teilnehmenden, die sich die Zeit genommen haben, an unserer Studie teilzunehmen. Wir danken Sophie Haesen und Arnaud 
Imber für ihren Einsatz in der Datenerhebung sowie der Datenanalyse. Wir danken der kanadischen Strafvollzugsbehörde „Correctional Service Canada" für die Unterstützung unserer Forschung durch die Rekrutierung von ExpertInnen. Wir danken Félix Pageau für die Unterstützung bei der Analyse der französischen Interviews.

Förderung Diese Arbeit wurde vom Schweizerischen Nationalfonds unterstützt [Förderungsnummer 166043] und von der Freiwilligen Akademischen Gesellschaft Basel.

Funding Open access funding provided by University of Basel

Interessenkonflikt H. Seaward, L. Taboas Hidalgo, T. Wangmo und B.S. Elger geben an, dass kein Interessenkonflikt besteht.

Open Access Dieser Artikel wird unter der Creative Commons Namensnennung 4.0 International Lizenz veröffentlicht, welche die Nutzung, Vervielfältigung, Bearbeitung, Verbreitung und Wiedergabe in jeglichem Medium und Format erlaubt, sofern Sie den/die ursprünglichen Autor(en) und die Quelle ordnungsgemäß nennen, einen Link zur Creative Commons Lizenz beifügen und angeben, ob Änderungen vorgenommen wurden.

Die in diesem Artikel enthaltenen Bilder und sonstiges Drittmaterial unterliegen ebenfalls der genannten Creative Commons Lizenz, sofern sich aus der Abbildungslegende nichts anderes ergibt. Sofern das betreffende Material nicht unter der genannten Creative Commons Lizenz steht und die betreffende Handlung nicht nach gesetzlichen Vorschriften erlaubt ist, ist für die oben aufgeführten Weiterverwendungen des Materials die Einwilligung des jeweiligen Rechteinhabers einzuholen.

Weitere Details zur Lizenz entnehmen Sie bitte der Lizenzinformation auf http://creativecommons.org/licenses/by/4.0/deed.de.

\section{Literatur}

\section{Verwendete Literatur}

Aebi M, Delgrande N (2013) Space-Facts \& figures. http://wp. unil.ch/space/files/2015/04/ENG_Executive-Summary_SPACE2013_150206.pdf. Zugegriffen: 19. Juli 2021

Al-Rousan T, Rubenstein L, Sieleni B, Deol H, Wallace RB (2017) Inside the nation's largest mental health institution: a prevalence study in a state prison system. BMC Public Health 17(1):342. https://doi.org/10.1186/s12889-017-4257-0

Baidawi S, Trotter C (2015) Psychological distress among older prisoners: a literature review. J Forensic Soc Work 5(1/3):234-257. https://doi.org/10.1080/1936928X.2015.1075166

Baidawi S, Trotter C (2016) Psychological distress among older prisoners: associations with health, health care utilization, and the prison environment. J Correct Health Care 22(4):354-366

Braun V, Clarke V (2006) Using thematic analysis in psychology. Qual Res Psychol 3(2):77-101. https://doi.org/10.1191/ 1478088706qp063oa

Bretschneider W, Elger BS (2014) Expert perspectives on Western European prison health services: do ageing prisoners receive equivalent care? Bioethical Inquiry 11(3):319-332

Bundesamt für Statistik (2020a) Massnahmenvollzug: Mittlerer Insassenbestand nach Geschlecht, Nationalität und Alter (je-d19.04.01.41) [Dataset]. https://www.bfs.admin.ch/bfs/de/home/ statistiken/kriminalitaet-strafrecht/justizvollzug.assetdetail.14817 448.html. Zugegriffen: 21. März 2017

Bundesamt für Statistik (2020b) Straf- und Massnahmenvollzug: Mittlerer Insassenbestand der über 49-jährigen Personen (je-d19.04.01.34) [Dataset]. https://www.bfs.admin.ch/bfs/de/home/ statistiken/kriminalitaet-strafrecht/justizvollzug/inhaftierte-erwac hsene.assetdetail.14817437.html. Zugegriffen: 19. März 2017

Council of Europe: Committee for the Prevention of Torture (2012) Rapport au Conseil fédéral suisse relatif à la visite effectuée en Suisse par le Comité européen pour la prévention de la torture et des peines ou traitements inhumains ou dégradants (CPT) du 10 au 20 octobre 2011. https://www.refworld.org/docid/5088f0f82. html. Zugegriffen: 19. Juli 2021

Davoren M, Fitzpatrick M, Caddow F, Caddow M, O'Neill C, O'Neill H, Kennedy HG (2015) Older men and older women remand prisoners: mental illness, physical illness, offending patterns and needs. Int Psychogeriatr 27(5):747-755

Di Lorito C, Völlm B, Dening T (2018) Psychiatric disorders among older prisoners: a systematic review and comparison study against older people in the community. Aging Ment Health 22(1):1-10. https://doi.org/10.1080/13607863.2017.1286453

Dietiker J, Dittmann V, Graf M (2007) Risk assessment of sex offenders in a German-speaking sample. Applicability of PCL-SV, HCR-20+ 3, and SVR-20. Nervenarzt 78(1):53-61

Dittmann V (2003) Was kann die Kriminalprognose heute leisten. In: Hässler F, Reberning E, Schnoor K (Hrsg) Forensische Kinder-, Jugend- und Erwachsenenpsychiatrie: Aspekte der forensischen Begutachtung. Schattauer, Stuttgart

Elger BS (2004) Prevalence, types and possible causes of insomnia in a Swiss remand prison. Eur J Epidemiol 19(7):665-677

Elger BS (2008) Towards equivalent health care of prisoners: European soft law and public health policy in Geneva. J Publ Health Policy 29(2):192-206. https://doi.org/10.1057/jphp.2008.6

Elger BS, Geohring C, Revaz SA, Morabia A (2002) Prescription of hypnotics and tranquilisers at the Geneva prison's outpatient service in comparison to an urban outpatient medical service. Soz Präventivmed 47(1):39-43. https://doi.org/10.1007/BF01318404

Elger BS, Handtke V, Wangmo T (2015) Informing patients about limits to confidentiality: a qualitative study in prisons. Int Law Psychiatry 41:50-57. https://doi.org/10.1016/j.ijlp.2015.03.007

Fazel S, Baillargeon J (2011) The health of prisoners. Lancet 377(9769): 956-965

Fazel S, Hope T, O’Donnell I, Piper M, Jacoby R (2001) Health of elderly male prisoners: worse than the general population, worse than younger prisoners. Age Ageing 30(5):403-407. https://doi. org/10.1093/ageing/30.5.403

Gates ML, Staples-Horne M, Walker V, Turney A (2017) Substance use disorders and related health problems in an aging offender population. J Health Care Poor Underserved 28(2):132-154

Glancy G (2006) Caveat Usare: actuarial schemes in real life. J Am Acad Psychiatry Law 34(3):272-275

Habermeyer E (2009) Der Nervenarzt. Forens Psychiatr 80(1):79-92

Habermeyer E, Passow D, Puhlmann P, Vohs K (2008) Preventive detention: empirical findings concerning the detainees and the quality of forensic-psychiatric reports. Fortschr Neurol Psychiatr 76(11):672-677

Habermeyer E, Passow D, Puhlmann P, Vohs K, Herpertz S (2009) Sexual offenders in preventive detention: data concerning the inmates and expert witness practice. Int J Offender Ther Comp Criminol 53(4):373-384

Haesen S, Merkt H, Imber A, Elger B, Wangmo T (2018) Substance use and other mental health disorders among older prisoners. Int J Law Psychiatry 62:20-31

Handtke V, Wangmo T, Elger B, Bretschneider WJ (2017) New guidance for an old problem: early release for seriously ill and elderly prisoners in Europe. Prison J 97(2):224-246

Howerton A, Byng R, Campbell J, Hess D, Owens C, Aitken P (2007) Understanding help seeking behaviour among male offenders: qualitative interview study. BMJ (Clinical research ed). https:// doi.org/10.1136/bmj.39059.594444.AE

Jang E, Canada KE (2014) New directions for the study of incarcerated older adults: Using social capital theory. J Gerontol Soc Work 57(8):858-871 
Kakoullis A, Le Mesurier N, Kingston P (2010) The mental health of older prisoners. Int Psychogeriatr 22(5):693-701. https://doi.org/ $10.1017 / \mathrm{S} 1041610210000359$

Kersten L, Cislo AM, Lynch M, Shea K, Trestman RL (2016) Evaluating START NOW: a skills-based psychotherapy for inmates of correctional systems. Psychiatr Serv 67(1):37-42

Kingston P, Le Mesurier N, Yorston G, Wardle S, Heath L (2011) Psychiatric morbidity in older prisoners: unrecognized and undertreated. Int Psychogeriatr 23(8): 1354-1360

Leigey ME, Hodge JP (2012) Gray matters: gender differences in the physical and mental health of older inmates. Women Crim Justice 22(4):289-308. https://doi.org/10.1080/08974454.2012.716358

Lewis CF, Fields C, Rainey E (2006) A Study of geriatric forensic evaluees: who are the violent elderly? J Am Acad Psychiatry Law 34(3):324-332

Långström N, Enebrink P, Laurén E-M, Lindblom J, Werkö S, Hanson RK (2013) Preventing sexual abusers of children from reoffending: systematic review of medical and psychological interventions. BMJ. https://doi.org/10.1136/bmj.f4630

Mercado CC, Ogloff JR (2007) Risk and the preventive detention of sex offenders in Australia and the United States. Int J Law Psychiatry 30(1):49-59

Merkt H, Haesen S, Meyer L, Kressig R, Elger B, Wangmo T (2020) Defining an age cut-off for older offenders: a systematic review of literature. Int J Prison Health 16(2):95-116. https://doi.org/10. 1108/IJPH-11-2019-0060

Merkt H, Wangmo T, Pageau F, Liebrenz M, Cornaz CD, Elger B (2020) Court-mandated patients' perspectives on the psychotherapist's dual loyalty conflict-Between ally and enemy. Front Psychol. https://doi.org/10.3389/fpsyg.2020.592638

Mokros A (2015) Standardisierte und psychometrische Untersuchungsverfahren in der forensisch-psychiatrischen Begutachtung. In: Psychiatrische Begutachtung. Elsevier, Amsterdam, S 29-52

Mokros A, Neumann CS, Stadtland C, Osterheider M, Nedopil N, Hare RD (2011) Assessing measurement invariance of PCL-R assessments from file reviews of North American and German offenders. Int J Law Psychiatry 34(1):56-63

Moschetti K, Stadelmann P, Wangmo T, Holly A, Bodenmann P, Wasserfallen J-B, Elger B, Gravier B (2015) Disease profiles of detainees in the Canton of Vaud in Switzerland: gender and age differences in substance abuse, mental health and chronic health conditions. BMC Public Health 15:872. https://doi.org/10.1186/ s12889-015-2211-6

Moschetti K, Zabrodina V, Wangmo T, Holly A, Wasserfallen J-B, Elger BS, Gravier B (2018) The determinants of individual health care expenditures in prison: evidence from Switzerland. BMC Health Serv Res 18(1):1-13

Riklin F (2014) Alt werden und Sterben hinter Gittern. Eine neue Realität für den Vollzug [Vieillir et mourir derrière les barreaux. Une nouvelle réalité de l'exécution des sanctions pénales]. Stämpfli, Bern

Rossegger A, Gerth J, Seewald K, Urbaniok F, Singh JP, Endrass J (2013) Current obstacles in replicating risk assessment findings: a systematic review of commonly used actuarial instruments. Behav Sci Law 31(1):154-164

Ruiz G, Wangmo T, Mutzenberg P, Sinclair J, Elger BS (2014) Understanding death in custody: a case for a comprehensive definition. J Bioeth Inq 11(3):387-398

SAMW (2002) Ausübung der ärztlichen Tätigkeit bei inhaftierten Personen. http://www.samw.ch/de/Ethik/Richtlinien/Aktuellgueltige-Richtlinien.html. Zugegriffen: 19. Juli 2021

Seaward H, Wangmo T, Egli-Alge M, Hiersemenzel L-P, Graf M, Elger BS, Habermeyer E (2021) Incarcerated older persons' motivation to engage in criminal court-mandated treatment: findings from a qualitative study. Forensic Sci Int Mind Law 2:100057

Sodhi-Berry N, Knuiman M, Alan J, Morgan VA, Preen DB (2015) Pre- and post-sentence mental health service use by a population cohort of older offenders ( $\geq 45$ years) in Western Australia. Soc Psychiatry Psychiatr Epidemiol 50(7):1097-1110
Spellman A, Heyne B (1989) Suicide? Accident? Predictable? Avoidable? The psychological autopsy in jail suicides. Psychiatr Q 60(2): 173-183

Trestman R, Appelbaum K, Metzner J (2015) Oxford textbook of correctional psychiatry. Oxford University Press, Oxford

Trestman RL, Eucker S, Müller-Isberner R (2007) The treatment of personality-disordered offenders in Germany. J Am Acad Psychiatry Law 35(2):229-234

Trestman RL, Ferguson W, Dickert J (2015) Behind bars: the compelling case for academic health centers partnering with correctional facilities. Acad Med 90(1):16-19

Trotter C, Baidawi S (2015) Older prisoners: challenges for inmates and prison management. Aust N Z J Criminol 48(2):200-218. https://doi.org/10.1177/0004865814530731

Turner M, Peacock M (2017) Palliative care in UK prisons: practical and emotional challenges for staff and fellow prisoners. J Correct Health Care 23(1):56-65

Wakai S, Shelton D, Trestman RL, Kesten K (2009) Conducting research in corrections: challenges and solutions. Behav Sci 27(5):743-752

Walmsley R (2013) World prison population list (tenth edition). International Centre for Prison Studies (ICPS). http://www. prisonstudies.org/resources/world-prison-population-list-10thedition. Zugegriffen: 19. Juli 2021

Walmsley R (2016) World prison population list. International Centre for Prison Studies, London

Wangmo T, Handtke V, Elger BS (2014) Disclosure of past crimes: an analysis of mental health professionals' attitudes towards breaching confidentiality. J Bioeth Inq 11(3):347-358. https://doi.org/ 10.1007/s11673-014-9546-z

Wangmo T, Meyer AH, Bretschneider W, Handtke V, Kressig RW, Gravier B, Bula C, Elger BS (2015) Ageing prisoners' disease burden: is being old a better predictor than time served in prison? Gerontology 61(2):116-123. https://doi.org/10.1159/000363766

Wangmo T, Meyer AH, Handtke V, Bretschneider W, Page J, Sommer J, Stuckelberger A, Aebi MF, Elger BS (2016) Aging prisoners in Switzerland: an analysis of their health care utilization. J Aging Health 28(3):481-502. https://doi.org/10.1177/ 0898264315594137

Wangmo T, Handtke V, Bretschneider W, Elger BS (2017) Prisons should mirror society: the debate on age-segregated housing for older prisoners. Ageing Soc 37(4):675-694

World Health Organisation (2014) Prisons and health. WHO Regional Office for Europe, Copenhagen

World Health Organization (2015) Fact sheet prison and health

Yarnell SC, Kirwin PD, Zonana HV (2017) Geriatrics and the legal system. J Am Acad Psychiatry Law 45(2):208-217

\section{Weiterführende Literatur}

Pageau F, Devaud Cornaz C, Gothuey I, Seaward H, Wangmo T, Elger B Prison downward spiraling of unhealthy lifestyle and poor mental health-A qualitative study. Under review

Pageau F, Seaward H, Habermeyer E, Elger B, Wangmo T Loneliness and social isolation among the older person in a Swiss secure institution: a qualitative study. Under review

Shaw D, Delacrausaz P, Aebi N, Elger B Definition of dangerousness. Under review

Shaw D, Merkt H, Pageau F, Wangmo T, Elger B Collective and other unjust punishment in Swiss prisons-A qualitative exploration. Under review

Wangmo T, Ruiz G, Sinclair J, Mangin P, Elger BS (2014) The investigation of deaths in custody: a qualitative analysis of problems and prospects. J Forensic Leg Med 25:30-37

Wangmo T, Seaward H, Pageau F, Hiersemenzel L-P, Elger B (2021) Forensic-psychiatric risk evaluations: perspectives of forensic psychiatric experts and older incarcerated persons from Switzerland. Front Psychiatry. https://doi.org/10.3389/fpsyt.2021.643096 